\title{
Tendencias investigativas que delimitan los campos de actuación del trabajo social en la gestión social ${ }^{1}$
}

\author{
Investigatory trends that define the fields of action of social \\ work in social management
}

Recibido: 16 de diciembre de 2014 - Revisado: 06 de febrero de 2015 - Aceptado: 06 de marzo de 2015

\section{Resumen}

Dado el creciente uso de la gestión social en el quehacer de diferentes disciplinas entre ellas el trabajo social, el presente artículo busca delimitar cuatro campos de actuación que le permitirán al Grupo de Investigación Innovación Social (Insol) de la Universidad Simón Bolívar (Cúcuta, Colombia), abordar de manera específica proyectos que profundicen en estos saberes. Se realizó una investigación cualitativa de corte documental, rastreando bases epistémicas de la gestión y el trabajo social; se indagaron además los grupos de investigación y programas académicos de trabajo social y ciencias sociales que relacionan la gestión social y se llegó a establecer como campos de actuación: la gestión para el desarrollo disciplinar; desarrollo y políticas; desarrollo, territorio, redes y organizaciones y gerencia, planificación y administración.

\section{Palabras clave}

Gestión social, trabajo social, campos de actuación.

\begin{abstract}
Given the increasing use of social management in the work of different disciplines including social work, this article seeks to define four fields of action that will enable the Group for Social Innovation (Insol- for its spanish acronym) Research at Universidad Simon Bolivar (Cúcuta, Colombia), specifically addressing projects to deepen this knowledge. A qualitative study of documentary type was made, tracing epistemic bases of management and social work; research groups and academic programs of social work and social sciences that relate social management and came to be established as fields of action are also inquired: development management discipline; development and policies; development, territory, networks and organizations and management, planning and administration.
\end{abstract}

\section{Keywords}

Social management, social work, fields of action.

\footnotetext{
${ }^{1}$ Artículo derivado de la investigación "Estado del arte de la línea de investigación 'Gestión para el desarrollo humano y organizacional"” del Grupo de Investigación Innovación Social (Insol) del Programa de Trabajo Social. Investigación financiada por la Universidad Simón Bolívar, sede Cúcuta (Colombia).

${ }^{2}$ Trabajadora social, Universidad Simón Bolívar, sede Cúcuta. Doctoranda en Educación, Universidad Pedagógica Experimental Libertador (Rubio, Venezuela). Magíster en Práctica Pedagógica, Universidad Francisco de Paula Santander (Cúcuta, Colombia). Especialista en Práctica Pedagógica Universitaria, Universidad Francisco de Paula Santander. Especialista en Gerencia Social, Universidad Simón Bolívar, sede Cúcuta. Coordinadora de investigación del Programa de Trabajo Social, Universidad Simón Bolívar, sede Cúcuta. Investigadora del Grupo Altos Estudios de Frontera. Correo electrónico:

c.ramirezm@unisimonbolivar.edu.co

Para citar este artículo use: Ramírez, C. (2016). Tendencias investigativas que delimitan los campos de actuación del trabajo social en la gestión social. Revista Civilizar Ciencias Sociales y Humanas, 16(30), 233-244.
} 


\section{Introducción}

En la actualidad diferentes disciplinas científicas de las ciencias sociales y humanas como la política, psicología, administración, derecho y trabajo social y múltiples posgrados vienen incorporando la gestión social como un campo de actuación complementario a sus perfiles, lo cual hace que la gestión social se vea cada vez más como algo difuso y abarcador. El trabajo social no es ajeno a esta tendencia que se asume para impactar en el desarrollo de procesos que permiten apoyarle en su objeto de estudio ${ }^{1}$.

Es así que se efectúa la presente investigación, para comprender el horizonte que la gestión social representa para el trabajo social, estableciendo con ello campos de actuación que le permitan al Grupo de Investigación Innovación Social (Insol) de la Universidad Simón Bolívar (Cúcuta, Colombia) direccionar sus estudios y proceder según las lógicas investigativas de Behar (2008), donde referencia:

La investigación científica es por su naturaleza un conocimiento de tipo instrumental, es un saber hacer con el conocimiento disciplinar para producir ideas-constructos nuevos, modelos teóricos, procesos de innovación, en definitiva, evidencia teórica y empírica que contribuya a una mejor comprensión de la realidad y facilite la detección y resolución de problemas concretos. En este sentido la investigación está siempre vinculada a la realidad, al campo de conocimiento disciplinar de aplicación, al contexto cultural, social y político en que se desarrolla y se convierte en la fuente de generación de pensamiento libre y útil, cuya difusión aproxima a científicos de diferentes campos disciplinares, enriquece la formación universitaria y orienta a actores sociales relevantes (p. 14).

Hecha la investigación se reportan tres hallazgos importantes. El primero de ellos es la transversalización que la gestión social ha realizado a los planes de estudio de trabajo social del país, ya que en la mayoría de estos se encuentra explícita en su perfil, currículo o misión educativa ${ }^{2}$. El segundo evidencia la gestión social como un creciente campo de estudio investigativo en el país, así lo muestran las siguientes cifras obtenidas en el rastreo de los GrupLAC $^{3}$, en donde se encontraron 1476 grupos de ciencias sociales, de los cuales 493 hacen énfasis en la gestión social, 154 de manera directa -con el nombre del grupo-y 339 con alguna de sus líneas de investigación ${ }^{4}$. El tercer hallazgo permite comprender que la gestión social dada su definición es una

[...] conjunción de varios campos científicos y disciplinas que contribuyen a definir su propósito, puesto que está dotada de un conjunto de elementos teórico-prácticos en función de transformar determinados aspectos de la realidad para el bienestar colectivo (Ruiz, 2008, párr. 1).

$\mathrm{Y}$ es este "transformar determinados aspectos de la realidad para el bienestar colectivo", lo que le coadyuva al trabajo social a sustentarse de manera específica en la gestión social para impulsar el desarrollo de la disciplina, las políticas públicas, el territorio, las redes, las organizaciones y la planificación social como aspectos fundamentales de su perfil profesional.

Es esta relevancia académica -asociada a documentos como La Visión 2019 (Departamento Nacional de Planeación, 2005), la política nacional de fomento a la investigación y la innovación, la política de innovación social en Colombia y los planes de desarrollo nacional, departamental y municipal que hacen hincapié en la necesidad de empoderamiento ciudadano y uso de la gestión social como estrategia de desarrollo- lo que hace aún más necesaria la definición de los campos de actuación del trabajo social en la gestión social, para iniciar su abordaje investigativo y de una forma focalizada, aportarle a la intervención disciplinar del programa académico en la Universidad Simón Bolívar. 


\section{Problema de investigación y metodología}

Ante la necesidad de consolidar la línea de gestión social para el desarrollo humano y organizacional del Grupo de Investigación Insol del Programa de Trabajo Social de la Universidad Simón Bolívar, urge el establecimiento de campos de actuación que delimiten estos estudios. Se busca fijar los límites en estos campos a fin de direccionar los procesos investigativos que permitan comprender los desarrollos del campo y con ello contribuir al quehacer del trabajo social en la región.

El estudio se desarrolló desde un enfoque cualitativo que se soportó en Mejía (2004), quien proporcionó las bases para el diseño de una revisión documental que hiciera posible comprender el recorrido histórico y la relación existente entre el trabajo social y la gestión, así como las visiones actuales sobre las que se deben generar nuevos campos de investigación y de actuación para la disciplina (p. 278).

La metodología se sustentó en la revisión crítica del conocimiento, alcanzada a partir de los estudios documentales, para ello se siguieron las indicaciones de Ruiz (2009) con la selección de textos, los cuales se agruparon según el aporte temático en: libros y documentos de trabajo social; libros y documentos sobre gestión social y páginas web (GrupLAC de Colciencias y programas de trabajo social), determinando una muestra de 13 textos de gestión y trabajo social, 493 grupos de investigación avalados y categorizados por Colciencias y 39 páginas web de programas académicos de trabajo social de Colombia y 6 páginas web de programas de trabajo social de América Latina (Argentina, Brasil, Chile, Costa Rica, México y Venezuela).

Para la recolección de la información se desarrollaron las siguientes acciones: acopio bibliográfico, lectura preliminar de los textos, delimitación de las categorías de análisis comunes para la interpretación de resultados, lectura minuciosa de la documentación lo que implica: reflexión e interpretación y su resultado fueron las ideas más importantes que se plasman en las fichas de contenido; organización de las fichas de contenido y revisión del esquema: su objetivo; valoración del material recopilado; localización de posibles lagunas y detección de excesos en las ideas transcritas. La posibilidad de revisar el esquema de trabajo con el fin de darle mayor orden y uniformidad, antes de redactar el borrador. Finalizada la recolección de datos, se procede a sistematizar, procesar, analizar e interpretar estos.

Para el análisis de información se siguió a Balestrini (1998) quien propone la revisión y lectura acuciosa de los contenidos a partir de un análisis global o lectura inicial, y luego una lectura rigurosa o comprensiva que permitió la extracción de los datos identificados. Una vez se concluyó el análisis de fichas de estudio se determinaron cuatro categorías -a) desarrollo disciplinar; b) desarrollo y políticas; c) desarrollo, territorio, redes y organizaciones y d) gerencia, planificación y administración- que permiten establecer los campos de actuación del trabajo social en la gestión social.

\section{Resultados}

El trabajo social es una profesión basada en la práctica y una disciplina académica que promueve el cambio y el desarrollo social, la cohesión social, y el fortalecimiento y la liberación de las personas. Los principios de la justicia social, los derechos humanos, la responsabilidad colectiva y el respeto a la diversidad son fundamentales para el trabajo social. Respaldada por las teorías del trabajo social, las ciencias sociales, las humanidades y los conocimientos indígenas, el trabajo social involucra a las personas y las estructuras para hacer frente a desafíos de la vida y aumentar el bienestar (Federación Internacional de Trabajo Social -FITS, 2014, p. 1).

Lo anterior ubica la profesión frente a unos desafíos constantes que le presenta el mismo contexto, que al ser dinámico y diverso 
le exigen hacer uso de la investigación para entender y apropiar necesidades y recursos que potencializan la transformación para un desarrollo integral de los habitantes. Es aquí donde surge la necesidad de crear grupos de investigación disciplinar que aúnen esfuerzos para comprender aspectos del desarrollo del trabajo social y las respectivas tendencias que se deben considerar para su impulso. Los grupos de investigación, tal como los define Colciencias (2014a, p. 59) se establecen según

[...] las grandes áreas de conocimiento definidas por la Organización para la Cooperación y el Desarrollo Económicos (OCDE) [...] en: 1. Ciencias agrícolas. 2. Ciencias médicas y de la salud. 3. Ciencias naturales. 4. Ciencias sociales. 5. Humanidades. 6. Ingeniería y tecnología.

Esta estructura además especifica que los grupos deben desarrollar sus planes de trabajo acorde con líneas de investigación, las cuales representan campos del conocimiento desde donde se organiza y sistematiza diversas teorías, metodologías y disciplinas que contribuyen "Al desarrollo del conocimiento, a la innovación tecnológica o a la solución de problemas cruciales" (Universidad Católica de Pereira, 2011, p. 7), por ello Insol encontró en la gestión social un espacio investigativo que permite la comprensión y el desarrollo de indagaciones que le aporten al trabajo social herramientas para su actuación.

Para la comprensión de la gestión social se tomará a Huergo como referente, quien aborda el concepto desde su etimología: gestus que traduce "actitud, gesto, movimiento del cuerpo" (Huergo, 2008, p. 1), algo habitual, cultural e internalizado por los sujetos y que lleva a una vivencia del mismo. Esta definición aunque amplia y precisa varía con el paso del tiempo, cuando se acuña la expresión latina gerere (conducir acciones), dando un significado de carácter activo, el cual aludió en un momento histórico, a la designación de estrategias de guerra en donde se organizaban la posición territorial, las fuerzas de los enemigos y al cómo derrotar su voluntad.

Un segundo significado de gestión social está concebido según el carácter cultural y activo de los pueblos: "acción artística, en cuanto creativa (y no repetitiva o meramente estructurada por recetas) que tiende a la gestación de procesos colectivos, con los otros (y no a pesar de los otros y de lo existente, o contra los otros)" (Huergo, 2008, p. 1).

La gestión social como acción lleva al término 'gestionar'. Según expresan Villamayor y Lamas (1998, citados por Oviedo-Betún, 2011) esta es una "acción integral, entendida como un proceso de trabajo y organización en el que se coordinan diferentes miradas, perspectivas y esfuerzos, para avanzar eficazmente hacia objetivos asumidos institucionalmente y que desearíamos que fueran adoptados de manera participativa y democrática" (p. 13). Esta acción involucra aspectos como toma de decisiones individuales y colectivas, que propenden por una mejor forma de actuación.

El concepto de gestión social es relacional con el trabajo social en la medida en que la disciplina propende por el "cambio y el desarrollo social, la cohesión social, y el fortalecimiento y la liberación de las personas, la justicia social, los derechos humanos, la responsabilidad colectiva y el respeto a la diversidad" (FITS, 2014, p. 1). Situaciones que exigen de la gestión social, ya que esta conjugación integral de campos y disciplinas le permiten al trabajo social definir elementos teórico-prácticos para abordar la realidad.

La forma en que la profesión se centra en las necesidades humanas determina su convicción de que la índole fundamental de esas necesidades exige que se satisfagan, y no por decisión personal, sino como un imperativo de justicia básica. Por ello, el trabajo social avanza hacia la consideración de los derechos humanos como el otro principio de organización de su práctica profesional. La transición de la orientación hacia las necesidades 
a la afirmación de los derechos se ha hecho imprescindible porque es preciso satisfacer las necesidades sustantivas tangibles. Una necesidad sustantiva puede traducirse en un derecho positivo equivalente y el goce de ese derecho es algo que se espera faciliten el Estado y entidades más allá del Estado Naciones Unidas (1995, p. 12).

La luchas por la inclusión, articulación de instituciones y mitigación de problemas sociales a través de una cultura política en la que se asume la gestión social con "grupos activistas que demandan y ejercen presiones para resolver problemas de la comunidad" (Chávez-Carapia, 2003, p. 83) es una muestra de la importancia de la gestión social como componente de dinamismo y desarrollo integral, el cual no se puede considerar un área más de actuación para el trabajador social, sino que le representa un campo del conocimiento integral a múltiples áreas y sectores de la sociedad y epistemológicos que el trabajo social ha venido abordando desde sus inicios.

\section{Los campos de actuación del trabajo social en la gestión social}

En atención a las concepciones de trabajo social y gestión social con las cuales se sustenta la trascendencia de la gestión en la disciplina del trabajo social, y estudiadas las tendencias de los programas académicos y de los grupos de investigación social, se lograron consolidar cuatro campos de actuación que se generan en la gestión social y que son para el trabajo social, un valioso horizonte de sentido si los estudia y apropia en la praxis.

\section{Campo 1. La gestión para el desa- rrollo disciplinar del trabajo social.}

Siendo la gestión un campo de actuación de diferentes disciplinas, se plantea hacer uso de ella para impulsar el desarrollo del trabajo social, el cual dada la dinámica de su objeto de estudio, le exige la mirada interna de su quehacer metodológico y bases epistémicas, para accionar en función de su crecimiento, la articu- lación holística, la fundamentación y el fortalecimiento que trae consigo la mirada introspectiva, crítica y científica que le permita trascender y dar respuesta a las necesidades existentes en el contexto que interviene. En la revisión documental se puede encontrar una tendencia amplia en las escuelas de trabajo social de América Latina y en Colombia, en la que se hace referencia a la necesidad que tiene el trabajo social de reconceptualizar constantemente sus cimientos epistémicos y metodológicos a fin de dar respuesta a los constantes cambios sociales.

Esta necesidad de revisión epistémica lleva a la concepción de la gestión social como un campo de acción relevante para el desarrollo disciplinar, que se aprecia en los discursos de las diferentes agremiaciones del trabajo social en el mundo y especialmente en Latinoamérica (véase tabla 1).

Tabla 1

Agremiaciones latinoamericanas del trabajo social

\begin{tabular}{|c|c|}
\hline Región/país & Agremiación \\
\hline $\begin{array}{l}\text { América } \\
\text { Latina }\end{array}$ & $\begin{array}{l}\text { Asociación Latinoamericana de Enseñanza e } \\
\text { Investigación en Trabajo Social }\end{array}$ \\
\hline Argentina & $\begin{array}{l}\text { - Federación Argentina de Asociaciones Profe- } \\
\text { sionales de Servicio Social } \\
\text { - Consejo Profesional de Graduados en Servicio } \\
\text { Social o Trabajo Social } \\
\text { - Colegio de Profesionales en Servicio Social de } \\
\text { la Provincia de Córdoba } \\
\text { - Colegio de Trabajadores Sociales del Rosario }\end{array}$ \\
\hline Brasil & $\begin{array}{l}\text { - Consejo Federal de Servicio Social } \\
\text { - Asociación Brasileña de Enseñanza e Investi- } \\
\text { gación en Servicio Social }\end{array}$ \\
\hline Chile & Colegio de Asistentes de Chile \\
\hline Colombia & $\begin{array}{l}\text { - Consejo Nacional de Trabajo Social } \\
\text { - Consejo Nacional de Educación en Trabajo } \\
\text { Social } \\
\text { - Asociación de Trabajadores Sociales del Mag- } \\
\text { dalena } \\
\text { - Asociación de Trabajadores Sociales del Valle }\end{array}$ \\
\hline Costa Rica & Colegio de Trabajadores Sociales de Costa Rica \\
\hline Perú & Colegio de Trabajadores del Perú \\
\hline Uruguay & Asociación de Asistentes Sociales del Uruguay \\
\hline
\end{tabular}

Fuente: Escuela de Trabajo Social, Universidad de Costa Rica, 2016. 
Los diversos temas que se destacan y hacen que el trabajo social piense su labor en los contextos actuales, exigen la revisión de: su epistemología, la producción teórica y los paradigmas emergentes en la disciplina, los métodos y metodologías de intervención, identidad, inter y transdisciplinariedad, la intervención social y las políticas públicas, áreas y campos de acción, la filosofía disciplinar y sus fundamentos conceptuales, las sistematizaciones de experiencias, los perfiles y el contexto, las asociaciones y los estudios complementarios.

Estas temáticas enmarcan el rol mismo de la academia en la sociedad, tal como lo describe Carrizo (s.f.) cuando ubica a la universidad como ente legitimador de producción de saberes, lo que lo compromete a gestionar el conocimiento, lo cual se debe iniciar con:

Las reformas universitarias en clave transdisciplinaria, el papel de los profesores y la formación de formadores en la estrategia de gestión social del conocimiento, la relación de las universidades con los medios de comunicación y las nuevas tecnologías de la información y la comunicación (p. 4).

Esta gestión requiere hacer debates acerca de:

La tensión dialéctica entre apropiación social y apropiación privada del conocimiento; Cambios en la ciencia que están incrementando la importancia de las tecnologías apropiables y por lo tanto la importancia de los temas de propiedad intelectual e industrial; La creación de lo público y procesos de aprendizaje social como dos componentes estratégicos de la apropiación social del conocimiento; El fomento a la innovación y a las organizaciones que aprenden; Intermediación y gestión del conocimiento como una función crítica en las sociedades del conocimiento (p. 4).

La gestión social le está demandando al trabajo social el cuestionamiento constante de su comprensión de sociedad, de sus posturas epistémicas y, sobre todo, de su accionar decisivo en el desarrollo de liderazgos que den respuesta a su esencia misma:
El trabajo social es una disciplina que pretende la transformación de una realidad social determinada, a través de un método científico propio, con el objetivo de modificarla, a fin de dar respuesta a las necesidades humanas de los hombres en la sociedad [...]. El trabajo social es una disciplina que tiene como objetivo no solo el conocimiento, sino que está orientada a la acción. Así es el proceso científico, el trabajo social persigue objetivos cognitivos -conocer y explicar-y los objetivos utilitarios prever y actuar (Escobar-Pérez \& SánchezMajada, 2000, p. 129).

\section{Campo 2. La gestión para el desarro- llo humano y las políticas sociales.}

Un segundo campo que reúne el mayor esfuerzo de investigación interdisciplinar hace referencia al desarrollo humano y las políticas sociales, en donde politólogos, sociólogos, economistas, ambientalistas, profesionales de la salud, ingenieros, entre otros, luchan por la integración social a partir de organizaciones de base, entidades no gubernamentales de promoción de derechos y emancipación ciudadana para lograr una participación activa en la disminución de la brecha social, generación de mayores oportunidades para sus habitantes y consecución de bienestar social mediante el diseño de políticas públicas que propendan por el desarrollo.

Molina (2012) resalta el campo de las políticas sociales en trabajo social, desde los años ochenta sin que las orientaciones de tal debate "hallan vinculado de manera explícita el tema de la administración/gerencia y con ello el sesgo en lo técnico-operativo" (p. 91). Para la autora, la gestión social:

Concitó el interés público y por lo tanto formó parte de la agenda de luchas, negociaciones y decisiones de actores sociales-políticos en el marco del Estado y la sociedad civil y dio lugar a leyes y/o política social y a organizaciones ejecutoras gubernamentales y no gubernamentales. La gerencia de organizaciones implica conducir la direccionalidad de la misma en la tensión de los intereses en conflicto (p. 93). 
Como referente de la gestión social al trabajo social, se destacan temáticas de investigación como: las políticas sociales, las teorías y propuestas de modernización económica y productiva, el desarrollo y los conflictos sociales, el desarrollo e impacto cultural, las instituciones sociales y sus transformaciones, el desarrollo integral y sectorial, la ciudadanía, los movimientos y organizaciones sociales, las identidades sociales, la demografía/población, el patrimonio cultural, las tendencias sociales, las tendencias en estratificación y clases sociales, la inclusión y exclusión social, los derechos humanos, el fortalecimiento democrático, el trabajo, economía y sociedad; el conflicto y desarrollo, la participación social y política, la cultura política, las políticas públicas y sociales, la administración y gestión de programas y servicios sociales, los servicios de bienestar para la población, la organización y desarrollo local y regional, la globalización, la cooperación internacional y el desarrollo.

\section{Campo 3. La gestión territorial, redes y organizaciones.}

Como una acción emergente de los procesos de globalización e intercambios económicos, culturales y políticos que se experimentan en el mundo, como la imposición de modelos de desarrollo neoliberal que afectan de manera directa el concepto de territorio, cultura, ambiente y dinámica interna de las personas y sociedades; se asume un compromiso por la identidad territorial y la protección de sus espacios ambientales y culturales; así como una lucha frontal por el trabajo digno que respete y valore la situación local de cada región. Lo cual genera la necesidad de trabajadores sociales críticos y gestores, según lo indica Ruiz (s.f.):

Gestores sociales especialistas en conocimientos técnicos de las problemáticas del sector que les toca atender, y con la suficiente formación, en cuanto a conocimientos y habilidades de gestión organizacionales y donde la planeación participativa convierte en elemento clave para generar encuentros, alianzas, redes que redimensionen los nuevos vínculos sociales y las nuevas formas de acción colectiva (p. 1).

Que conciban una planificación territorial participativa en la que se tenga en cuenta la diversidad de los actores, sus intereses y poderes con el objeto de lograr incidencia directa en acciones que beneficien al colectivo, por ello en este campo del conocimiento se encuentran temáticas como desarrollo local, desarrollo territorial, ciudad-región, organización territorial, comunas, juntas de acción local-comunal, ciudad-visión rural, zonas de reserva campesinaindígena, parques naturales, vida social emergente, subculturas, tendencias ocupacionales, evaluación de tecnologías, el futuro del trabajo, movimientos sociales y asociacionismo, cultura de la empresa, condiciones de trabajo y seguridad de los trabajadores, estrés laboral, seguridad social, gestión del talento humano y responsabilidad social organizacional.

Es un campo que genera un compromiso de estudio de las dinámicas sociales que están repercutiendo en crecimientos acelerados de los barrios, las transformaciones de uso del suelo y la visión cambiante de territorio que llama a una lucha directa por la inclusión de sectores menos favorecidos, los cuales son víctimas de las dinámicas segregadoras que se reflejan en carentes oportunidades propiciadas por las instituciones locales, lo que llama a un urgente trabajo en función de redes y tecnologías innovadoras para la sociedad. Es por ello que este campo necesita de gestión social para indagar sobre la generación de conocimiento que impulse el empoderamiento y desarrollo territorial, que abandona el asistencialismo y se hace propositivo de su progreso.

\section{Campo 4. La gerencia, planifica- ción y administración.}

Este último campo de acción se enmarca en una visión de gerencia social que vincula de manera directa a empresas del Estado, organi- 
zaciones de base, organizaciones no gubernamentales y comunidades, que requieren articularse de forma activa en la planificación para la toma de decisiones e incidencia directa en los cambios sociales requeridos.

Se encontraron temáticas específicas como: diseño, ejecución, control y evaluación de planes, programas y proyectos de desarrollo humano y social, gerencia de lo público y marketing social. En este sentido se resalta a González y Rodríguez (2014), quienes han descrito la gestión social:

El management o gestión, nacida de la administración empresarial se ha convertido en el eje de cambio en las instituciones. Todo proceso posible de ser identificado, clasificado y estudiado tiene que ser gerenciado. La excelencia, la calidad total, el logro del cero defecto, los círculos de calidad, la planificación por proyecto, la autonomía, el liderazgo, la innovación, hacen parte de los aspectos que forman la gestión. Esta constituye más que una simple forma de planificación o administración: involucra un cambio en el modo en que pensamos, actuamos, sentimos; en suma, un cambio en la forma de ser de los individuos y de las organizaciones. La gestión aparece como una forma de hacer frente a la nueva realidad donde la libertad y la autonomía hacen parte de la sociedad de la información y del conocimiento (p. 3).

Este análisis evidencia la importancia de la gestión social para el trabajo social en cada uno de los campos descritos, pues estos son el resultado de prácticas que se vienen dando, por ello clasificarlos y agruparlos en estas categorías permitirá su estudio focalizado, la articulación directa con la gestión social y sobre todo, tener resultados que van a influir en el desarrollo social, situación que se alcanza como meta y efecto de estas dos disciplinas.

\section{Discusión}

Conforme con lo referenciado por Ruiz (2008) y citado a lo largo del texto, la gestión social es una "conjunción de varios campos científicos y disciplinas que contribuyen a definir su propósito" (el bienestar colectivo); por tanto, no debe hacerse uso de la gestión social como un concepto tan general o intangible que pierda su funcionalidad para el trabajo social, sino que al abordarlo desde los cuatro campos aquí estructurados se abren espacios de investigación disciplinar focalizada, la cual da respuesta a las tendencias de la sociedad actual y articula la gestión del conocimiento para que la universidad dé respuesta a las demandas sociales actuales. La gestión social según Ruiz (2008) permite el desarrollo de un perfil, en el que sobresalen atributos como:

[...] capacidad de coordinación y de negociación dentro de su propia organización y fuera de ella con otras organizaciones e instituciones, capacidad de administrar la contingencia, la complejidad y la incertidumbre, capacidad de dirigir estrategias de conducción participativas, con respecto al propio personal y a las personas de los programas y proyectos sociales $[\ldots]$.

Que sean artífices para mejorar la calidad de vida de sus comunidades, que promuevan la participación y la organización de sus comunidades para reconstruir el tejido social en la búsqueda de una sociedad más equilibrada, que optimicen y aumenten los recursos existentes en el medio para potenciar el desarrollo de sus comunidades.

Conviva y trabaje en sociedad solidariamente, participe en el diagnóstico de su realidad identificando sus problemas y planteando las soluciones más acordes a su realidad, elabore planes y proyectos para mejorar sus condiciones de vida, intervenga en la solución de los problemas detectados, tenga conocimiento e información sobre las actividades, programas y proyectos que el Estado emprenda en un territorio específico, facilite la interrelación, comunicación y negociación con el Estado y con otras organizaciones, maneje y distribuya los recursos humanos, técnicos y financieros de manera más eficiente y controle, fiscalice y vigile la acción del Estado y sus funcionarios (pp. 1-2, 5). 
Estos atributos son los que llevan incidir en el desarrollo humano o desarrollo de una sociedad que goza de bienestar, situación que demuestra la estrecha relación entre gestión social y trabajo social, ya que:

La intervención en el trabajo social es entendida como la acción organizada y desarrollada por los trabajadores sociales con las personas, grupos y comunidades. Sus objetivos están orientados a superar los obstáculos que impiden avanzar en el desarrollo humano y en la mejora de la calidad de vida de la ciudadanía. Intervención profesional que se basa en los fundamentos éticos, epistemológicos y metodológicos del trabajo social, desde un enfoque global, plural y de calidad. Estos planteamientos del trabajo social parten de una concepción dinámica de la persona que se inscribe en un contexto social, histórico, político, económico y cultural de la realidad social, conectando con otras disciplinas y profesionales (Barranco, 2004, p. 79).

Por ello se destaca -tras la revisión documental- una serie de autores que se sitúan en las tesis del desarrollo humano y que abordan de manera teórica los cuatro campos del trabajo social, gestión social que aquí se ha estructurado y que representa horizontes teóricos para ser apropiados en el Grupo de Investigación Insol desde la línea de gestión para el desarrollo humano y organizacional.

Tabla 2

Teóricos del desarrollo humano desde una perspectiva sociocrítica

\begin{tabular}{|c|c|}
\hline \multicolumn{2}{|c|}{ Visión paradigmática: el desarrollo humano desde perspectivas sociocríticas } \\
\hline \multicolumn{2}{|r|}{ Teóricos } \\
\hline Manfred Max Neef & Eduardo Gudynas \\
\hline Amartya Sen & Ana María Larrea \\
\hline Martha Nussbaum & Raúl Prada Alcoreza \\
\hline Carlo Tassara & Michael L. Ross \\
\hline Debenulin Severine & Jean Ziegler \\
\hline Ingrid Robeyns & Eduardo Galeano \\
\hline Tomás Rodríguez Villasante & Sistema de las Naciones Unidas \\
\hline Carlos Lozares Colina & Programa de las Naciones Unidas para el Desarrollo \\
\hline Alberto Acosta & Comisión Económica para América Latina y el Caribe \\
\hline Mario Blaser & Instituto Latinoamericano y del Caribe de Planificación Económica y Social \\
\hline Boaventura De Sousa Santos & División de Desarrollo Sostenible de las Naciones Unidas \\
\hline José de Echave & \\
\hline Arturo Escobar & \\
\hline Norberto Alayón & \\
\hline Antonio Vázquez Barquero & \\
\hline
\end{tabular}

Fuente: elaboración propia.

El Grupo de Investigación Insol del Programa de Trabajo Social de la Universidad Simón Bolívar, sede Cúcuta (Colombia), con el desarrollo y fundamentación de su línea de investigación a partir de estos cuatro campos de conocimiento, optimiza esfuerzos para dar respuesta a la política nacional de fomento a la investigación y la innovación, cuando hace referencia que la gestión para el desarrollo debe

[...] construir las condiciones más favorables para que el país se desarrolle integralmente en lo social, lo económico, lo político y lo cultural para ello pone de relieve la importancia de la ciencia, la tecnología y la innovación bajo los siguientes objetivos específicos: incrementar la generación de conocimiento, fomentar la innovación y el desarrollo productivo, fomentar la apropiación social de la CTI en la sociedad colombiana, incrementar y fortalecer las capacidades humanas para CTI, consolidar la institucionalidad del SNCTI, consolidar la infraestructura y los sistemas de información para la CTI, promover la integración regional, consolidar la proyección 
internacional de la CTI. 1. Apoyo a la formación avanzada de investigadores, 2. Consolidación de capacidades para CTI, incluye: (apoyo a la generación de conocimiento y fortalecimiento institucional de agentes del SCNTI), 3. Transformación productiva, mediante el fomento a la innovación y el desarrollo productivo, 4. Consolidación institucional del SNCTI, incluye: (fortalecimiento de sistemas de información e indicadores de CTI), fomento a la apropiación social de la CTI en la sociedad colombiana, dimensiones regional e internacional (Colciencias, 2014b, p. 69).

Política que concuerda con otros planes de orden internacional como los objetivos de desarrollo sostenible, La Visión 2019, el Conpes 3582: política nacional de ciencia, tecnología e innovación, así como los planes de orden nacional, departamental, regional, municipal $\mathrm{y}$ diversas iniciativas que han resaltado la necesidad de trabajar de manera conjunta en pro de articular la investigación a los temas sociales que requieren un cambio y que encuentran en la gestión social/trabajo social, un espacio de análisis que les provea las herramientas para su comprensión y abordaje asertivo.

\section{Conclusiones}

Se destaca la gestión social como espacio disciplinar-científico que le proporciona al trabajo social las bases teóricas, conceptuales y estratégicas necesarias para abordar campos del conocimiento que son intrínsecos a su quehacer. Dada su relevancia y amplitud, se identificaron tendencias de trabajo en los grupos de investigación del país y escuelas de trabajo social en Colombia y América Latina, que llevaron a la concreción de cuatro campos del conocimiento que permitirán el acercamiento de temas investigativos acordes con el contexto actual, como fueron: a) la gestión para el desarrollo disciplinar del trabajo social, b) el desarrollo humano y políticas sociales, c) desarrollo, territorio y redes organizacionales y d) gerencia, planificación y administración.

\section{Notas}

1 El trabajo social comparte con las ciencias sociales el mismo objeto material (dupla ser humano/sociedad) pero es el objeto formal (perspectiva desde la que se ocupa del objeto) lo que diferencia una ciencia de otra. "La definición del objeto tiene que ser abstracta y además del qué tiene que incorporar a quién y el dónde" (Zamanillo, 1999, p. 25, Citado por Calvo-Rojas, 2010, p. 2), en este caso es importante el uso de la gestión social para alcanzar el desarrollo humano y social promovido por los trabajadores sociales.

2 Sistema Nacional de Información de la Educación Superior. Búsqueda de programas de educación superior, que registra 84 programas con registro calificado en el país, los cuales fueron objeto de análisis en la presente investigación. Consultado en: http://snies.mineducacion. gov.co/consultasnies/programa\#

${ }^{3}$ GrupLAC (Grupo Latinoamérica y del Caribe), es un software originalmente desarrollado por el Grupo Stela de la Universidad Federal de Santa Catarina, cuyo objetivo es mantener un directorio de los grupos de investigación, instituciones e investigadores que participan activamente en el desarrollo de nuevas estrategias en el ámbito de la Ciencia, la Tecnología y la Innovación. Gracias a la Convocatoria de Grupos Colombianos de Investigación de 2002, el Grupo Académico CT\&S-UN, de la Universidad Nacional de Colombia, desarrolló la versión en línea para Colciencias en el 2003, la cual dispone de varias mejoras relacionadas con: producción científica del grupo basada en las hojas de vida de sus integrantes, pertenencia a más de una institución, relaciones con empresas, reportes de revisión de información, entre otras. En consecuencia, la colección sistematizada del conocimiento, la experiencia y la producción científica de todos los grupos colombianos que participan en actividades de investigación, innovación y desarrollo tecnológico, se encuentra disponible en la Internet. 
(Universidad Pedagógica y Tecnológica de Colombia. 2016. En línea)

${ }^{4}$ Datos obtenidos en el desarrollo de la investigación.

\section{Referencias}

Balestrini-Acuña M. (1998). Estudios documentales, teóricos, análisis de discurso y las historias de vida. Una propuesta metodológica para la elaboración de sus proyectos. Caracas: Servicio Editorial Consultores Asociados.

Barranco-Expósito, C. (2004). La intervención en trabajo social desde la calidad integrada. Alternativas. Cuardenos de Trbajo Social, 12, 79-102. Recuperado de http://rua. ua.es/dspace/bitstream/10045/5592/1/ ALT_12_05.pdf

Behar, D. S. (2008). Metodología de la investigación. Recuperado de http://museoarqueologico.univalle.edu.co/imagenes/ Proyecto\%20de\%20Grado\%201/lecturas/Libro\%20metodologia\%20investigacion.\%20Libro\%20NB.pdf

Calvo-Rojas, V.(2010). Elobjeto de estudio en Trabajo Social: una visión desde el psicoanálisis, la ley y la estructura. Portularia, 10(2), 9-20. Recuperado de http://google.redalyc. org/articulo.oa?id=161015612002

Carrizo, L. (s.f). Gestión social del conocimiento: Un nuevo contrato entre Universidad y sociedad. Recuperado de https://arquitecturaurbanismoinnovacion.files.wordpress.com/2013/07/gestic3b3n-socialdel-conocimiento.pdf

Chávez-Carapia, J. C. (2003). Participación social retos y perspectivas. México: UNAM.

Consejo Nacional de Política Económica y Social -[CONPES]. (27 de Abril de 2009).
Documento Conpes 3582. Recuperado de http://www.uelbosque.edu.co/sites/default/files/pdf/investigaciones/documento_compes_ciencia_tecnologia_3582.pdf

Departamento Administrativo de Ciencia, Tecnología e Innovación -[Colciencias]. (2014a). Modelo de medición de grupos de investigación, desarrollo tecnológico o de innovación y de reconocimiento de investigadores del sistema nacional de ciencia, tecnología e innovación. Recuperado de http://www. urosario.edu.co/Home/investigacion/Documentos/Documento-Modelo-MedicionGrupos-(1)/

Departamento Administrativo de Ciencia, Tecnología e Innovación -[Colciencias]. (2014b). Política nacional de fomento a la investigación y la innovación. Recuperado de http://www.colciencias.gov.co/ centro_recursos/colombia-construye-ysiembra-futuro-pol-tica-nacional-defomento-la-investigaci-n-y-

Departamento Administrativo de Ciencia, Tecnología e Innovación -[Colciencias]. (2014c). Ciencia y tecnología para todos. Recuperado de http://scienti.colciencias. gov.co:8083/ciencia-war/BusquedaGrupoXArea.do;jsessionid=82EAB9DCEB06 2690BAE344F637A73293

Departamento nacional de Planeación. (2005). Visión Colombia II centenario: 2019. Recuperado de http://archivo.cepal.org/ pdfs/GuiaProspectiva/visionColombiaIIc entenario_2019comple.pdf

Escobar-Pérez, C., \& Sánchez-Majada, G. (2000). El Trabajo Social en la era de la incertidumbre. Salamanca, España: Universidad de Salamanca.

Federación Internacional de Trabajo Social [FITS]. (2014). Definición global del trabajo social aprobada en la asamblea 
general de la FITS Federación Internacional de Trabajo Social. Recuperado de http://www.adasu.org/prod/1/487/Definicion.Global.del.Trabajo.Social..pdf

González, J., Rodríguez, Y. (2014). Marco epistemológico de la gestión. Posibilidades pedagógicas en la educación superior (Congreso Iberoamericano de Ciencia, Tecnología, Innovación y Educación). Recuperado de http://www.oei.es/congreso2014/memoriactei/828.pdf

Huergo, J. (2008). Los procesos de gestión. Recuperado de http://servicios2.abc.gov.ar/ lainstitucion/univpedagogica/especializaciones/seminario/materialesparadescargar/seminario4/huergo3.pdf

Mejía-Navarrete, J. (2004). Sobre la investigación cualitativa. Nuevos conceptos y campos de desarrollo. Lima: Editorial Unms.

Molina-Molina, M. L. (2012). La gestión de la política social en el debate del trabajo social crítico. En, Gestión social para el desarrollo humano (pp. 91-122). Bogotá: Editorial Universidad Colegio Mayor de Cundinamarca. Recuperado de http:// unicolmayor.edu.co/recursos_user/portal/ rec/arc_4907.pdf

Naciones Unidas. (1995). Serie de capacitación profesional $N^{\circ} 1$ derechos humanos $y$ trabajo social, manual para escuelas de servicio social y trabajadores sociales profesionales. Nueva York; Ginebra: Autor. Recuperado de http://cdn.ifsw.org/ assets/ifsw_104630-7.pdf

Oviedo-Betún, M. I. (2011). Gestión, liderazgoy valores en la administración de la unidad educativa fisco misional experimental compensatoria a distancia "Dr. Camilo Gallegos Dominguez" de macas-morona Santiago durante el periodo lectivo 2010 -
2011. (Tesis de Maestría). Universidad Católica de Lojas, Loja, Ecuador. Recuperado de http://dspace.utpl.edu.ec/ bitstream/123456789/6773/1/Tesis\%20 APROBADA.pdf

Política de Innovación Social. (2014). Construyamos la política de innovación social. Recuperado de https://somosmas.org/ project/construyamos-la-politica-deinnovacion-social/

Ruiz, K. (s.f.). Teoría y práctica de la gestión social. Recuperado de http://www.semahn.chiapas.gob.mx/portal/descargas/ guardaparques/articulos/Textos_Teoria_ prctica_de_gestion_social.pdf

Ruiz, K. (2008). Teoría y práctica de la gestión social I. Recuperado de http:// cienciassocialeskathy.obolog.com/teoriapractica-gestion-social-i-568642

Ruiz-Ruiz, J. (2009). Análisis sociológico del discurso: métodos y lógicas. Forum: Qualitative Social Research, 10(2). Recuperado de http://www.qualitativeresearch.net/index.php/fqs/article/ view/1298/2776

Universidad Católica de Pereira. (2011). Guía para institucionalizar: Líneas de investigación Proyectos de investigación Grupos de investigación Semilleros de investigación. Consultado en: http://temporal.ucp.edu.co/ ucp_docs/dii/dii_guia_institucionalizar_ investigacion.pdf

Universidad Pedagógica y Tecnológica de Colombia. (2016). ¿Qué es el GrupLac? Recuperado de http://www.uptc.edu.co/ direccion_investigaciones/enlaces/p_8

Universidad de Costa Rica. (2016). Sitios de trabajo social en América Latina. Recuperado de http://www.ts.ucr.ac.cr/int/america.php 